\title{
Compression Angle of Ossification of the Posterior Longitudinal Ligament and Its Clinical Significance in Cervical Myelopathy
}

\author{
Nam Lee, M.D., ${ }^{1}$ Do Heum Yoon, M.D., Ph.D., ${ }^{1}$ Keung Nyun Kim, M.D., Ph.D., ${ }^{1}$ Hyun Chul Shin, M.D., Ph.D., ${ }^{2}$ Dong Ah Shin, M.D., Ph.D., ${ }^{1}$ \\ Yoon Ha, M.D., Ph.D. ${ }^{1}$ \\ Department of Neurosurgery, ${ }^{1}$ Spine and Spinal Cord Institute, Yonsei University College of Medicine, Seoul, Korea \\ Department of Neurosurgery, ${ }^{2}$ Kangbuk Samsung Hospital, Sungkyunkwan University School of Medicine, Seoul, Korea
}

Objectives : The correction of clinical and radiologic abnormalities in patients with symptomatic ossification of the posterior longitudinal ligament (OPLL) is the current mainstay of treatment. This study aimed to identify radiographic predictors of severity of myelopathy in patients with symptomatic OPLL.

Methods : Fifty patients with symptomatic cervical OPLL were enrolled. Based on Japanese Orthopedic Association (JOA) scores, patients were divided into either the mild myelopathy $(n=31)$ or severe myelopathy $(n=19)$ group. All subjects underwent preoperative plain cervical roentgenogram, computed tomography (CT), and MR imaging (MRI). Radiological parameters (C2-7 sagittal vertical axis, SVA; C2-7 Cobb angle; C2-7 range of motion, ROM; OPLL occupying ratio; and compression angle) were compared. Compression angle of OPLL was defined as the angle between the cranial and caudal surfaces of OPLL at the maximum level of cord compression

Results : The occupying ratio of the spinal canal, C2-7 Cobb angle, C2-7 SVA, types of OPLL, and C2-7 ROM of the cervical spine were not statistically different between the two groups. However, the OPLL compression angle was significantly greater $(p=0.003)$ in the severe myelopathy group than in the mild myelopathy group and was inversely correlated with JOA score $(r=-0.533, p<0.01)$. Furthermore, multivariate regression analysis demonstrated that the compression angle $(B=-0.069, p<0.001)$ was significantly associated with JOA scores $(R=0.647, p<0.005)$. Conclusion : Higher compression angles of OPLL have deleterious effects on the spinal cord and decrease preoperative JOA scores.

Key Words : Ossification of the posterior longitudinal ligament · Cervical myelopathy · Compression angle.

\section{INTRODUCTION}

Ossification of the posterior longitudinal ligament (OPLL) involves replacement of ligamentous soft tissue with new ectopic bone $^{16)}$. Cervical spine OPLL can cause neurological deficits secondary to myelopathy due to the compression of neural elements in the spinal canal. Left untreated, myelopathy due to OPLL can cause irreversible damage. It is therefore important to identify the variables responsible for OPLL induction of cervical myelopathy and to develop an objective measure for predicting the severity of myelopathy in patients with symptomatic cervical OPLL as a way to augment their treatment.

Many investigations have been conducted to elucidate the factors that affect myelopathy severity in OPLL disease. Those reports considered various factors to be predictors of myelopa- thy, including the occupying ratio (diameter of OPLL occupying the spinal canal to the unoccupied diameter of the spinal canal) ${ }^{17)}$, Pavlov ratio (anterior-posterior vertebral body diameter to the spinal canal diameter $)^{10)}$, percent range of motion (ROM) (ROM at the intervertebral disc level responsible for myelopathy divided by total ROM multiplied by 100$)^{8)}$, segmental ROM (ROM at the maximum level of cord compression based on flexion and extension radiographs ${ }^{5)}$, axial ossified pattern of OPLL (laterally deviated or centrally located) ${ }^{7}$, and C2-7 total ROM (maximal flexion angle subtracted by maximal extension angle $)^{15)}$. While each of these studies show substantial results using the aforementioned parameters, it is difficult to identify which clinical factor best predicts disease severity because these parameters are so closely correlated with each other.

The aim of this study is to investigate which radiological pa-

- Received : January 20, $2016 \cdot$ Revised : May 30, $2016 \cdot$ Accepted : June 1, 2016

- Address for reprints : Yoon Ha, M.D., Ph.D.

Department of Neurosurgery, Spine and Spinal Cord Institute, Yonsei University College of Medicine, 50-1 Yonsei-ro, Seodaemun-gu, Seoul 03722, Korea

Tel : +82-2-2228-2165, Fax : +82-2-393-9979, E-mail : hayoon@yuhs.ac

- This is an Open Access article distributed under the terms of the Creative Commons Attribution Non-Commercial License (http://creativecommons.org/licenses/by-nc/3.0) which permits unrestricted non-commercial use, distribution, and reproduction in any medium, provided the original work is properly cited. 
rameters best correlate with myelopathy severity.

\section{MATERIALS AND METHODS}

\section{Patient population}

Institutional review board approval was obtained before initiating this study.

Fifty patients with symptomatic OPLL of the cervical spine were retrospectively reviewed. All patients were consecutively consulted at our institution between April 2012 and November 2013. The patient cohort included 40 men and 10 women, with ages ranging from 42 to 72 years (Table 1). All patients enrolled in this study had axial neck pain, arm pain due to radiculopathy or sensory/motor deficit, gait disturbance, and/or urinary symptoms due to myelopathy. A total of 50 patients were to undergo surgery for posterior decompression.

Table 1. Modified Japanese Orthopedic Association (JOA) score for assessment of cervical myelopathy

Score
Category

I. Motor dysfunction of the upper extremity

Unable to handle both spoon and chopstick

Unable to handle chopsticks, able to eat with a spoon

Able to handle chopsticks with much difficulty

Able to handle chopsticks with slight difficulty

None

II. Motor dysfunction of the lower extremity

Unable to walk

Can walk on flat floor with walking aid

Can walk up and/or down stairs with handrail or walking aid

Lack of stability and smooth gait

None

III. Sensory deficit of the upper extremity

Definite sensory loss

Slight sensory loss

None

IV. Sensory deficit of the lower extremity

Definite sensory loss

Slight sensory loss

None

V. Sensory deficit of the trunk

Definite sensory loss

Slight sensory loss

None

VI. Bladder function deficit

Unable to void

Able to void with voiding procedure

Able to void with severe effort (retention)

Able to void with mild effort

Void with frequency

Void with hesitation

None

The JOA score in a healthy subject is the total of the best scores : $(1++1++\|++\mathrm{V}+\mathrm{V}+$ $\mathrm{VI})=20$ points

\section{Neurological evaluation}

Neurological outcomes were assessed using the visual analogue scale (VAS), neck disability index (NDI), short form health survey (SF-36) and arm-trunk-leg-bladder-scores of the Japanese Orthopedic Association Score (JOA score). The JOA score system used in our institute was modified so that a score of 20 meant no myelopathy, i.e., a healthy subject (Table 1). Patients were divided into either the mild myelopathy group (JOA score $\geq 17, \mathrm{n}=31$ ) or the severe myelopathy group (JOA score $\leq 16, \mathrm{n}=$ 19 ). The aforementioned indices were analyzed for both groups.

\section{Radiographic measurements}

Overall lordotic alignment between the $\mathrm{C} 2-7$ vertebrae was measured using the $\mathrm{C} 2-7 \mathrm{Cobb}$ angle method on lateral X-ray, C2-7 sagittal vertical axis, C2-7 total ROM, and sagittal alignment (Fig. 1) ${ }^{15)}$. The C2-7 Cobb angle is defined as the angle of intersection between lines parallel to the inferior end plates of C2 and C7, while the C2-7 sagittal vertical axis (SVA) is defined as the deviation of the $\mathrm{C} 2$ plumb line from the posterior superior endplate of $\mathrm{C}^{12)}$. Patients were stratified into a kyphosis group $\left(\mathrm{C} 2-7 \mathrm{Cobb}\right.$ angle $\left.<0^{\circ}, \mathrm{n}=12\right)$, straight group $\left(0^{\circ} \leq \mathrm{C} 2-7\right.$ Cobb angle $\left.<10^{\circ}, \mathrm{n}=16\right)$ and lordosis group $(\mathrm{C} 2-7 \mathrm{Cobb}$ angle $\geq 10^{\circ}, \mathrm{n}=22$ ) by their sagittal alignment using lateral cervical radiographs (Table 2). The number of levels involving OPLL, type of OPLL and the occupying ratio of the spinal canal were evaluated with computed tomography (CT). Morphologically, patients were classified as having continuous $(n=8)$, segmental $(n=16)$, mixed $(n=20)$ or localized $(n=6)$ OPLL types according to criteria of the Japanese Investigation Committee on Ossification of the Spinal Ligaments (Table 2) ${ }^{17)}$. High signal intensity (SI) change on T2-weighted MRI, vertical length of SI change in the spinal cord, and angle of compression at the maximal compressed level were examined.

The compression angle is a special localized OPLL angle at the maximal compression level on sagittal view of $\mathrm{T} 2$ weighted
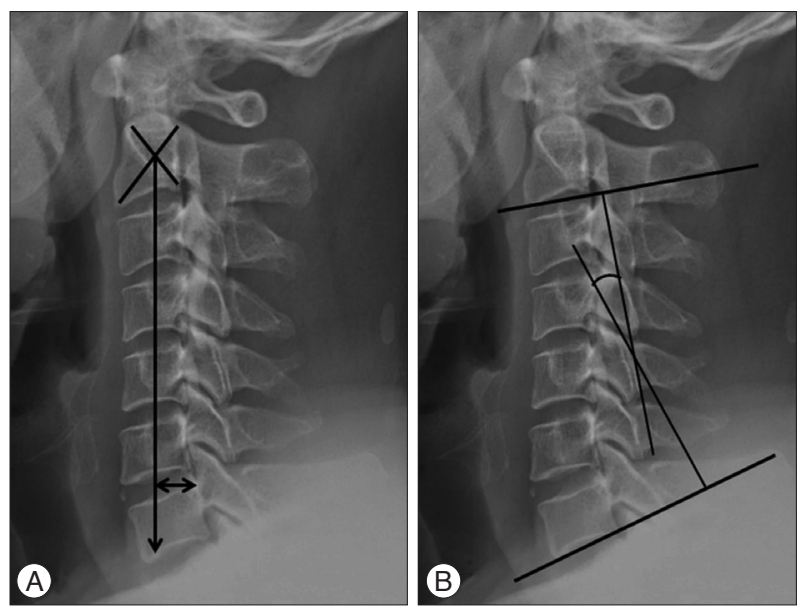

Fig. 1. A : Measurement of C2-7 SVA interval between $\mathrm{C} 2$ body plumb line and posterior superior endplate of C7. B : Sagittal alignment by C2-7 Cobb angle method : angle between two lines parallel to the endplate of $\mathrm{C} 2$ and $\mathrm{C} 7$ on lateral plain radiographs. 
MRI. The angle was measured using two lines extended to the peak point of the OPLL mass, one drawn from the cranial surface of the OPLL and the other drawn from the caudal surface of the OPLL (Fig. 2). The angle made by the intersection of these

Table 2. Clinical characteristics of cervical OPLL

\begin{tabular}{ll}
\hline No. of patients (number) & 50 \\
Gender (M: F) & $40: 10$ \\
Age (years) & $42-72$ (mean 57.2) \\
OPLL type $^{\star}(\mathrm{n})$ & \\
$\quad$ Continuous type & 8 \\
$\quad$ Segmental type & 16 \\
$\quad$ Mixed type & 20 \\
$\quad$ Localized type & 6 \\
Sagittal alignment ${ }^{\dagger}(\mathrm{n})$ & \\
$\quad$ Kyphosis & 12 \\
$\quad$ Straight & 16 \\
$\quad$ Lordosis & 22 \\
Degree of compression angle & (n) \\
$\quad$ Low angle group & \\
$\quad$ High angle group & 26
\end{tabular}

${ }^{*}$ Classification by Investigation Committee on ossification of posterior longitudinal ligaments (OPLLS) of the Japanese Ministry of Public Health and Welfare, ${ }^{\dagger}$ Classification by $\mathrm{C} 2-7 \mathrm{Cobb}$ angle. Kyphosis $<0^{\circ}, 0^{\circ} \leq$ straight $<10^{\circ}, 10^{\circ} \leq$ Lordosis, ${ }^{\ddagger}$ Classification by compression angle. Low angle group $<50^{\circ}$, high angle group $\geq 50^{\circ}$

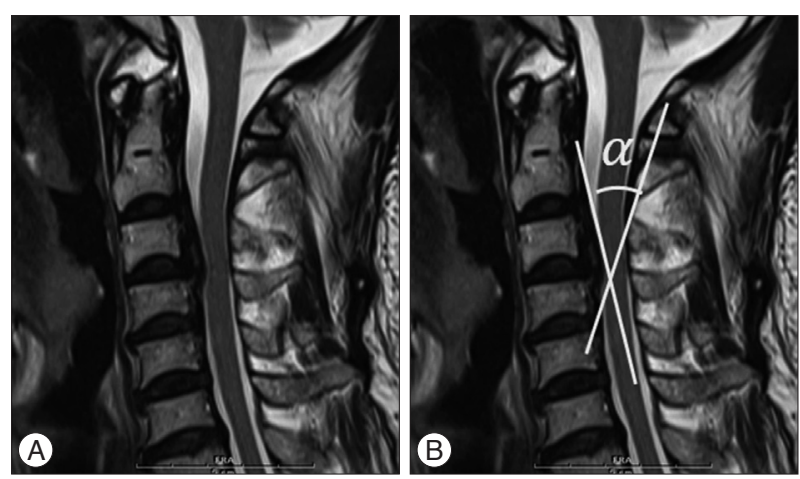

Fig. 2. Compression angle of OPLL. Special kyphotic angle at most compressed level. In this study, the angle was measured by sagittal T2 weighted preoperative MRI image. A : $\mathrm{C} 3 / 4$ is the most compressed level on T2WI. B : We measured the compression angle at C3/4 level. a : compression angle. two lines represents the OPLL angle. If there are more than two different compressed levels, we measured compression angle at the level which showed high signal intensity of spinal cord on MRI. Based on OPLL angle, patients were divided into either a low angle group (compression angle $<50^{\circ}, \mathrm{n}=26$ ) or a high angle group (compression angle $\geq 50^{\circ}, \mathrm{n}=24$ ) (Table 2). Clinical outcomes were then compared between the two angle groups.

\section{Validation of the measurement}

To achieve the reliability of the measurement, 2 examiners ( 1 clinical fellow and 1clinical associate professor) measured all parameters in 50 cases 2 times with 1 week interval. Inter-examiner and intra-examiner ICC and 95\% CI were assessed. Interexaminer reliability was assessed by the intraclass correlation coefficient (ICC) of data obtained for the measurement of each parameter among all two examiners. In addition, intra-examiner reliability was also assessed between the first and second measurements by two examiners.

\section{Statistical analysis}

The independent t-test was applied to compare differences between the two groups (compression angle relative to disease severity score). Measured variables were analyzed using multiple regression analysis (age, sex, C2-7 SVA, Cobb angle, ROM, number of levels in which involving OPLL, occupying ratio, compression angle to JOA score) and correlation analysis (age, sex, C2-7 SVA, Cobb angle, ROM, number of levels involving OPLL, occupying ratio, compression angle relative to disease severity score). Categorical variables were analyzed using ANOVA (age, sex, C2-7 SVA, Cobb angle, ROM, number of levels involving OPLL, occupying ratio, compression angle relative to OPLL type, and clinical outcomes score relative to sagittal alignment) with the PASW Statistic 18 software program. A $p$ value less than 0.05 was considered statistically significant.

\section{RESULTS}

Pearson correlation analysis was used to identify which parameters affected other parameters as well as which parameters

Table 3. Factors affecting clinical outcomes

\begin{tabular}{|c|c|c|c|c|c|}
\hline & VAS-neck & VAS-arm & NDI & SF-36 & JOA score \\
\hline & $\mathrm{r} / p$ value & $\mathrm{r} / p$ value & $\mathrm{r} / p$ value & $\mathrm{r} / p$ value & $\mathrm{r} / p$ value \\
\hline Age & $0.09 / 0.52$ & $0.34 /<0.05$ & $0.25 / 0.07$ & $-0.12 / 0.39$ & $0.02 / 0.88$ \\
\hline Gender & $0.02 / 0.84$ & $0.004 / 0.97$ & $-0.12 / 0.37$ & $0.15 / 0.29$ & $-0.04 / 0.76$ \\
\hline C2-7 SVA (mm) & $-0.15 / 0.28$ & $-0.14 / 0.33$ & $-0.09 / 0.50$ & $0.01 / 0.90$ & $-0.09 / 0.51$ \\
\hline C2-7 Cobb angle $\left(^{\circ}\right)$ & $0.00 / 1.00$ & $-0.26 / 0.06$ & $-0.05 / 0.72$ & $0.07 / 0.60$ & $-0.03 / 0.79$ \\
\hline $\mathrm{C} 2-7 \mathrm{ROM}\left(^{\circ}\right)$ & $-0.04 / 0.77$ & $-0.06 / 0.67$ & $0.06 / 0.66$ & $0.03 / 0.79$ & $0.17 / 0.22$ \\
\hline No. of OPLL involved & $-0.02 / 0.88$ & $-0.11 / 0.43$ & $-0.17 / 0.22$ & $0.07 / 0.60$ & $-0.04 / 0.76$ \\
\hline Occupying ratio & $-0.01 / 0.95$ & $0.09 / 0.52$ & $0.19 / 0.17$ & $0.05 / 0.72$ & $-0.14 / 0.33$ \\
\hline Compression angle $\left(^{\circ}\right)$ & $-0.06 / 0.64$ & $-0.19 / 0.17$ & $0.05 / 0.70$ & $-0.17 / 0.23$ & $-0.53 /<0.001$ \\
\hline
\end{tabular}

Correlation analysis was performed to analyze correlation between parameters and clinical outcomes. $r$ : Pearson correlation coefficient, VAS : visual analogue scale, NDI : neck disability index, SF-36 : 36-Item Short Form Health Survey Instrument, JOA : Japanese Orthopedic Association, SVA : sagittal vertical axis, ROM : range of motion, $\mathrm{OPLL}$ : ossification of the posterior longitudinal ligament 
Table 4. Effects of radiological parameters on JOA score

\begin{tabular}{|c|c|c|c|c|}
\hline & \multicolumn{2}{|c|}{ Unstandardized coefficient } & \multirow{2}{*}{$\begin{array}{c}\text { Standardized coefficient } \\
\text { Beta }\end{array}$} & \multirow{2}{*}{$p$ value } \\
\hline & B & SE & & \\
\hline A constant & 15.033 & 2.966 & & 0.000 \\
\hline Gender & 0.342 & 0.639 & 0.072 & 0.595 \\
\hline Age & 0.045 & 0.035 & 0.194 & 0.212 \\
\hline C2-7 SVA (mm) & -0.014 & 0.020 & -0.100 & 0.481 \\
\hline C2-7 Cobb angle $\left(^{\circ}\right)$ & -0.024 & 0.026 & -0.146 & 0.352 \\
\hline No. of OPLL involved & 0.461 & 0.317 & 0.200 & 0.154 \\
\hline Occupying ratio & -2.257 & 1.749 & -0.176 & 0.204 \\
\hline Compression angle $\left(^{\circ}\right)$ & -0.069 & 0.017 & -0.521 & $<0.001$ \\
\hline SI change on MRI & -0.921 & 0.504 & -0.242 & 0.075 \\
\hline
\end{tabular}

Multiple regression testing was performed to analyze correlation between se, age, C2-7 SVA, C2-7 Cobb angle, no. of OPLL involved, occupying ratio, compression angle, SI change on MRI and JOA score. SE : standard error of the means, JOA : Japanese Orthopedic Association, SVA : sagittal vertical axis, OPLL : ossification of the posterior longitudinal ligament, $\mathrm{SI}$ : signal intensity

Table 5. Factors affecting severity of myelopathy

\begin{tabular}{|c|c|c|c|}
\hline & $\begin{array}{l}\text { Mild myelopathy } \\
\qquad(\mathrm{n}=31)\end{array}$ & $\begin{array}{l}\text { Severe myelopathy } \\
\qquad(\mathrm{n}=19)\end{array}$ & $p$ \\
\hline & Mean \pm SE & Mean \pm SE & \\
\hline C2-7 SVA (mm) & $22.49 \pm 2.53$ & $23.83 \pm 3.08$ & 0.743 \\
\hline C2-7 Cobb angle $\left(^{\circ}\right)$ & $8.81 \pm 2.20$ & $10.21 \pm 2.42$ & 0.681 \\
\hline $\mathrm{C} 2-7 \mathrm{ROM}\left(^{\circ}\right)$ & $38.19 \pm 1.93$ & $32.00 \pm 2.80$ & 0.067 \\
\hline Occupying ratio & $0.43 \pm 0.02$ & $0.46 \pm 0.03$ & 0.480 \\
\hline No. of OPLL involved & $4.77 \pm 0.15$ & $4.68 \pm 0.18$ & 0.713 \\
\hline Compression angle $\left({ }^{\circ}\right)$ & $44.47 \pm 2.27$ & $56.66 \pm 3.30$ & $<0.01$ \\
\hline
\end{tabular}

Independent t-test. SVA : sagittal vertical axis, ROM : range of motion, Compression ratio : compression ratio of spinal canal by OPLL, Compression angle : kyphosis angle of OPLL at the maximum cord compression level, SE : standard error of the means

Table 6. Comparison of clinical outcomes between two types

\begin{tabular}{lcccrr}
\hline & $\begin{array}{c}\text { Low angle group } \\
(\mathrm{n}=26)\end{array}$ & & $\begin{array}{c}\text { High angle group } \\
(\mathrm{n}=24)\end{array}$ & \\
\cline { 2 - 2 } \cline { 5 - 6 } & Mean \pm SE & & Mean \pm ve & \\
\hline NDI & $10.81 \pm 1.71$ & & $13.54 \pm 1.53$ & 0.243 \\
SF-36 & $107.27 \pm 2.16$ & & $102.33 \pm 1.96$ & 0.100 \\
JOA score & $17.96 \pm 0.30$ & & $16.21 \pm 0.38$ & $<0.001$ \\
\hline
\end{tabular}

Independent t-test. ${ }^{*}$ Classification by compression angle. Low angle $<50^{\circ}$, high angle $\geq 50^{\circ}$. SE : standard error of the means, NDI : Neck Disability Index, SF-36 : 36-Item Short Form Health Survey Instrument, JOA : Japanese Orthopedic Association

best predicted clinical outcomes. Only the compression angle showed significant correlation with JOA score (Table 3). Therefore, multiple regression analysis was conducted to further clarify the effect compression angle had on JOA score. Gender, age, C2-7 SVA, C2-7 Cobb angle, number of levels involving OPLL, occupying ratio did not correlate with JOA scores, while only the compression angle did, and SI change on MRI tended to decrease the JOA score (Table 4). In patients with mild myelopathy (JOA score $\geq 17, \mathrm{n}=31$ ), the average compression angle was $44.47^{\circ}$ (range, $15.4^{\circ}-64.9^{\circ}$ ) while for those with severe myelopathy (JOA score $\leq 16, \mathrm{n}=19$ ), the average compression angle was $56.66^{\circ}$ (range, $30.2^{\circ}-85.8^{\circ}$ ). Other parameters, including $\mathrm{C} 2-7$ SVA, C2-7 Cobb angle, ROM, occupying ratio, and the number of levels involving OPLL, were not significantly different between those with mild and severe myelopathy (Table 5).

Compression angle groups were defined as low or high. With regard to clinical outcomes, JOA score was significantly higher in the low angle group than in the high angle group (17.96 vs. $16.21, p<0.001)$. However, NDI and SF-36 were not significantly different between these two groups (Table 6).

\section{Comparison of radiological findings with OPLL types}

ANOVA analysis was conducted according to OPLL classification by type (2). With regard to occupying ratio, segmental OPLL exhibited smaller parameters than other types $(0.32, p<$ 0.001). Other parameters, including C2-7 SVA, C2-7 Cobb angle, ROM and compression angle, did not show any differences among OPLL types (Table 7).

\section{Comparison of clinical outcomes with sagittal alignment}

Using sagittal alignment, fifty patients were stratified into the kyphosis, straight, or lordosis groups. ANOVA analysis was conducted for sagittal alignment and clinical outcomes. Only the VAS-arm score was statistically different and the kyphosis group had a higher VAS-arm score than the straight and lordosis groups (5.33 vs. 3.50 and $2.59, p<0.05$ ). However, others clinical parameters (VAS-neck, NDI, and SF-36) were not significantly different for each group (Table 8).

\section{DISCUSSION}

Our data suggests that high compression angle is associated with severe myelopathy and results in deteriorating JOA scores (Fig. 3). Using analysis to identify the relationship between various parameters and clinical outcomes, our findings implied that compression angle has a substantial relationship with JOA score. Age, gender, C2-7 SVA, C2-7 Cobb angle, C2-7 ROM, number of levels involving OPLL and occupying ratio do not have significant relationships with any clinical outcome scales in present study. Using multiple regression analysis and controlling confounding variables, we concluded that compression angle has a 
Table 7. Comparison of parameters between OPLL types*

\begin{tabular}{|c|c|c|c|c|c|}
\hline & Continuous type $(\mathrm{n}=8)$ & Segmental type $(\mathrm{n}=16)$ & Mixed type $(n=20)$ & Localized type $(n=6)$ & מ מ \\
\hline & Mean \pm SE & Mean \pm SE & Mean \pm SE & Mean \pm SE & $p$ value \\
\hline C2-7 SVA (mm) & $28.17 \pm 6.35$ & $16.58 \pm 3.10$ & $24.70 \pm 2.68$ & $27.58 \pm 5.43$ & 0.131 \\
\hline C2-7 Cobb angle $\left(^{\circ}\right)$ & $9.90 \pm 3.21$ & $8.14 \pm 2.95$ & $12.12 \pm 2.52$ & $2.53 \pm 5.74$ & 0.338 \\
\hline $\mathrm{C} 2-7 \mathrm{ROM}\left({ }^{\circ}\right)$ & $29.07 \pm 4.02$ & $37.11 \pm 2.88$ & $38.29 \pm 2.62$ & $33.30 \pm 4.35$ & 0.259 \\
\hline Occupying ratio & $0.50 \pm 0.03$ & $0.32 \pm 0.04$ & $0.49 \pm 0.02$ & $0.48 \pm 0.04$ & $<0.001$ \\
\hline Compression angle $\left({ }^{\circ}\right)$ & $50.10 \pm 4.06$ & $49.41 \pm 3.02$ & $49.20 \pm 4.27$ & $46.65 \pm 2.42$ & 0.976 \\
\hline
\end{tabular}

${ }^{*}$ Classification by the investigation committee on ossification of posterior longitudinal ligaments (OPLL) of the Japanese Ministry of Public Health and Welfare. SE : standard error of the means, SVA : sagittal vertical axis, ROM : range of motion

Table 8. Comparison of clinical outcomes with sagittal alignment*

\begin{tabular}{|c|c|c|c|c|}
\hline & Kyphosis group $(\mathrm{n}=12)$ & Straight group $(\mathrm{n}=16)$ & Lordosis group $(\mathrm{n}=22)$ & \\
\hline & Mean \pm SE & Mean \pm SE & Mean \pm SE & $p$ value \\
\hline VAS-neck & $2.58 \pm 0.75$ & $3.50 \pm 0.68$ & $2.45 \pm 0.55$ & 0.465 \\
\hline VAS-arm & $5.33 \pm 0.89$ & $3.50 \pm 0.82$ & $2.59 \pm 0.52$ & $<0.05$ \\
\hline NDI & $13.00 \pm 1.74$ & $14.50 \pm 2.25$ & $9.91 \pm 1.78$ & 0.217 \\
\hline SF-36 & $102.42 \pm 3.47$ & $104.56 \pm 2.63$ & $106.50 \pm 2.13$ & 0.565 \\
\hline JOA score & $17.25 \pm 0.52$ & $17.06 \pm 0.58$ & $17.09 \pm 1.68$ & 0.965 \\
\hline
\end{tabular}

${ }^{*}$ Classification by $\mathrm{C} 2-7 \mathrm{Cobb}$ angle. Kyphosis $<0^{\circ}, 0^{\circ} \leq \mathrm{Straight}<10^{\circ}, 10^{\circ} \leq$ Lordosis. SE : standard error of the means, NDI : Neck Disability Index, SF-36 : 36 -Item Short Form Health Survey Instrument, VAS : visual analogue scale, JOA : Japanese Orthopedic Association

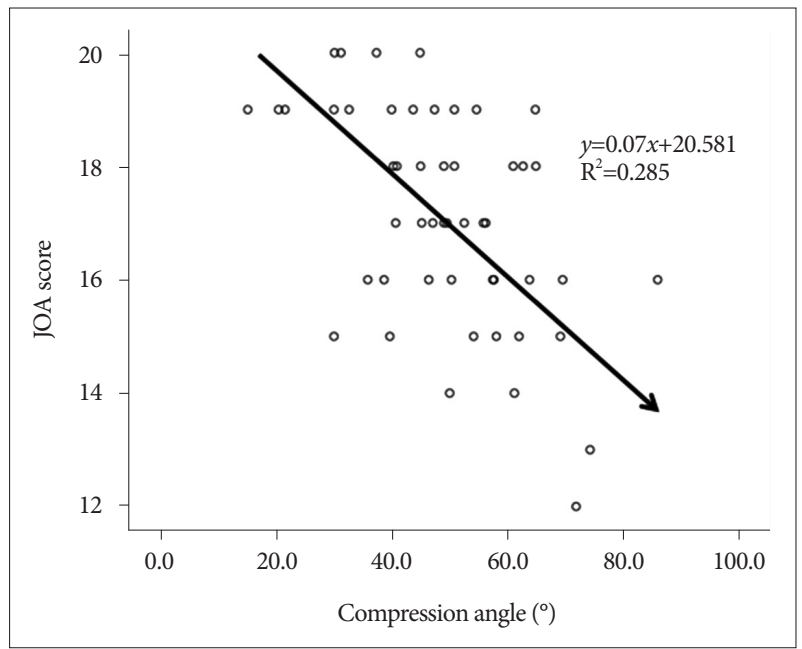

Fig. 3. Regression analysis shows negative correlation for compression angle and JOA score. JOA : Japanese Orthopedic Association.

significant inverse effect on JOA score. In addition, only compression angle was significantly different between the mild myelopathy group and severe myelopathy group. The compression angle is a specialized local kyphotic angle of OPLL mass itself. For every patient, we confirmed a maximum compressed level in the cervical spine using sagittal T2 weighted images of preoperative MRI and this level is often concordant with high signal intensity changed level. From that level, we measured the compression angle using two lines extended to the peak point of the OPLL mass, one line drawn from the cranial surface of the OPLL and the other drawn from the caudal surface of the OPLL. Therefore, the sharp and kyphoticpeak shapes of OPLL can have higher compression angles. Of these, the OPLL was generally measured from lateral plain radiograph or the sagittal view of
CT scan. However, the compression angle can reflect soft tissue of PLL as well as OPLL or the status of spinal cord compression, so we believe that MRI is better than CT scan in present study ${ }^{14)}$.

Previous studies reported kyphosis as an important risk factor contributing to worse clinical outcomes, especially JOA score $^{1,11)}$. Taniyama et al. ${ }^{13)}$ emphasized that local kyphosis contributes more to clinical outcomes than general kyphosis, such as C2-7 Cobb angle or sagittal alignment. They suggested that while preoperative global kyphosis did not affect clinical outcomes, the minimum interval between the tip of local kyphosis and a line connecting the midpoint of the cord at the level of the inferior endplates of C2 and C7 did have an effect.

Based on previous studies, we hypothesized that the compression angle is a significant contributor in determining severity of myelopathy. When the compression angle is high, the force compressing the spinal cord is greater than with a low angle. In these situations, the compression angle of OPLL can act as a tension force on the spinal cord. This force increases more with neck flexion than neck extension. It causes mechanical compression to the ventral portion of the spinal cord. As a result, spinal cord ischemia can occur. Although a small area of the OPLL mass compresses the ventral portion of the spinal cord, this tension force can interrupt the flow of the anterior spinal artery or CSF. This is an important pathophysiological factor in developing myelopathy ${ }^{6}$. In this study, the mean occupying ratio of all enrolled patients was 0.44 (range $0.12-0.77$ ). The compression angle is meaningful because it implies that in most cases, the tip of the OPLL mass and the ventral portion of the spinal cord have close adhesion without a visible gap. Tokuhashi et al. ${ }^{14)}$ reported that ossification-kyphosis angle had a statistically significant relationship with certain parameters in an echo-free space study. When echo-free space between the OPLL and spinal cord was 
absent on intraoperative ultrasonography, the ossification-kyphosis angle was higher than when echo-free space was preserved. Oshima et al..$^{9)}$ described the segmental kyphotic angle as an adverse prognostic factor for patients with myelopathy. In this manner, there are many articles that emphasize the importance of local kyphosis.

Various factors should be considered when estimating the severity of myelopathy in OPLL. Previous studies suggested that static factors such as occupying ratio and Pavlov ratio could help to determine disease severity ${ }^{7,8,14}$. Other studies concluded that dynamic factors such as segmental ROM have a significant effect on determining disease severity ${ }^{3}$. Recent studies focused on sagittal alignment in the cervical region emphasize that sagittal alignment including C2-7 SVA was a substantial factor in determining clinical outcomes ${ }^{2,12,18}$. However, we did not find any significant correlation between referenced parameters and clinical outcomes in this study.

Consideration is needed regarding why these factors did not show any significant results in this study. First, the occupying ratio revealed different affects on myelopathy in OPLL between the two groups. The mild myelopathy group showed smaller occupying ratio values than the severe myelopathy group. However, there was no statistically significant difference ( 0.43 vs. $0.46, p$ value $=0.480$ ). This discrepancy might be caused by the ossified pattern of OPLL lesions, which can be divided into a lateral deviated type and a central localized type in axial view. Matsunaga et al.") described the lateral deviated type of OPLL as a substantial risk factor for myelopathy. This type can be asymptomatic when the OPLL is located centrally with a certain occupying ratio, but the lateral deviated type can also induce severe symptoms such as myelopathy.

The C2-7 ROM measured in this study also did not show a statistically significant difference between the two groups (38.1 vs. 32.0 , $p$ value $=0.067$ ). Fujiyoshi et al. ${ }^{3)}$ reported that segmental ROM significantly affects myelopathy development in patients with cervical OPLL. Segmental ROM was measured at the maximum cord compression level composed of one adjacent segment from flexion and extension radiographs. However, we measured C2-7 global ROM rather than segmental ROM, and we did not observe significant results for this parameter in our study. We can consider these unexpected results limitations of this study. Another limitation is in regard to measurement of the compression angle. Most previous studies conducted to clarify risk factors for myelopathy severity used plain X-ray or 3DCT imaging tools. Studies using MRI as an imaging tool have not been reported. Similar to our measurement methods, Tokuhashi et al. ${ }^{14)}$ measured the ossification-kyphosis angle of the decompression site from the sagittal view of preoperative MRIs. They suggested that the ossification-kyphosis angle affects clinical outcomes. However, this concept of ossification-kyphosis angle is different from that of the compression angle. Ossificationkyphosis angles contain several sites decompressed by surgery without reflecting the shape of OPLL at the site with most com- pression. On the contrary, compression angle is measured at just the most compressed level. Round and dome shaped OPLLs have a flat surface at the compression site while compression angles are close to zero degrees. The compression angle was not measured or excluded from this study when other masses compressed the spinal cord posteriorly, such as ossification of the ligamentumflavum or facet joint hypertrophy. In that situation, the spinal cord can be compressed not only ventrally, but also dorsally and laterally.

For patients with segmental type OPLL, the occupying ratio was substantially lower. Although this study does not suggest that classification by type of OPLL affects clinical outcomes, Fujiyoshi et al. ${ }^{3)}$ suggested that segmental type OPLL has lower occupying ratios than other OPLL types. Because segmental-type OPLL has larger segmental ROM as a dynamic factor, it can cause neurologic symptoms even with lower occupying ratios. Because segmental type OPLL can have larger C2-7 ROM than other OPLL types, any neck motion can induce impingement injury to the spinal cord. In continuous type OPLL, patients usually have smaller C2-7 ROM than in other OPLL types, reducing the possibility of impingement injury.

Cervical radiculopathy is a common condition resulting from compression of the neural foramen ${ }^{14)}$. VAS-arm is considered representative of radiculopathy severity. The lordosis group had C2-7 Cobb angles greater than 10 degrees and a lower VAS-arm score than other groups. In the normal anatomical spine, the cervical spine has a mild lordotic curve. Therefore, it is reasonable that this group shows a lower VAS-arm score ${ }^{4)}$.

\section{CONCLUSION}

The compression angle might be a novel method related to the severity of myelopathy. If compression angle is high, myelopathy occurs more often. If the compression angle is low, there is reduced possibility of myelopathy. Compression angles on sagittal view MRI may provide useful information to surgeons in predicting the severity of myelopathy for patients with OPLL. When evaluating severity of myelopathy, the compression angle might be another parameter to evaluate or predict the clinical outcomes.

\section{- Acknowledgements}

This work was supported by the 2013 Korea Health Technology R\&D Project, Ministry of Health \& Welfare, Republic of Korea (A120254).

\section{References}

1. Chiba K, Ogawa Y, Ishii K, Takaishi H, Nakamura M, Maruiwa H, et al. : Long-term results of expansive open-door laminoplasty for cervical myelopathy--average 14-year follow-up study. Spine (Phila Pa 1976) 31 : 2998-3005, 2006

2. Ferch RD, Shad A, Cadoux-Hudson TA, Teddy PJ : Anterior correction of cervical kyphotic deformity : effects on myelopathy, neck pain, and sagittal alignment. J Neurosurg 100 : 13-19, 2004

3. Fujiyoshi T, Yamazaki M, Okawa A, Kawabe J, Hayashi K, Endo T, et al. : 
Static versus dynamic factors for the development of myelopathy in patients with cervical ossification of the posterior longitudinal ligament. J Clin Neurosci 17 : 320-324, 2010

4. Gore DR, Sepic SB, Gardner GM : Roentgenographic findings of the cervical spine in asymptomatic people. Spine (Phila Pa 1976) 11 : 521524, 1986

5. Masaki Y, Yamazaki M, Okawa A, Aramomi M, Hashimoto M, Koda M, et al. : An analysis of factors causing poor surgical outcome in patients with cervical myelopathy due to ossification of the posterior longitudinal ligament : anterior decompression with spinal fusion versus laminoplasty. J Spinal Disord Tech 20 : 7-13, 2007

6. Masson C, Leys D, Meder JF, Dousset V, Pruvo JP : [Spinal cord ischemia]. J Neuroradiol 31 : 35-46, 2004

7. Matsunaga S, Nakamura K, Seichi A, Yokoyama T, Toh S, Ichimura S, et al. : Radiographic predictors for the development of myelopathy in patients with ossification of the posterior longitudinal ligament : a multicenter cohort study. Spine (Phila Pa 1976) 33 : 2648-2650, 2008

8. Morio Y, Nagashima H, Teshima R, Nawata K : Radiological pathogenesis of cervical myelopathy in 60 consecutive patients with cervical ossification of the posterior longitudinal ligament. Spinal Cord 37 : 853-857, 1999

9. Oshima Y, Seichi A, Takeshita K, Chikuda H, Ono T, Baba S, et al. : Natural course and prognostic factors in patients with mild cervical spondylotic myelopathy with increased signal intensity on T2-weighted magnetic resonance imaging. Spine (Phila Pa 1976) 37 : 1909-1913, 2012

10. Pavlov H, Torg JS, Robie B, Jahre C : Cervical spinal stenosis : determina- tion with vertebral body ratio method. Radiology $164: 771-775,1987$

11. Suda K, Abumi K, Ito M, Shono Y, Kaneda K, Fujiya M : Local kyphosis reduces surgical outcomes of expansive open-door laminoplasty for cervical spondylotic myelopathy. Spine (Phila Pa 1976) 28 : 1258-1262, 2003

12. Tang JA, Scheer JK, Smith JS, Deviren V, Bess S, Hart RA, et al. : The impact of standing regional cervical sagittal alignment on outcomes in posterior cervical fusion surgery. Neurosurgery 76 suppl 1 : S14-S21, 2015

13. Taniyama T, Hirai T, Yamada T, Yuasa M, Enomoto M, Yoshii T, et al. : Modified K-line in magnetic resonance imaging predicts insufficient decompression of cervical laminoplasty. Spine (Phila Pa 1976) 38 : 496501, 2013

14. Tokuhashi $\mathrm{Y}$, Matsuzaki H, Oda H, Uei H : Effectiveness of posterior decompression for patients with ossification of the posterior longitudinal ligament in the thoracic spine : usefulness of the ossification-kyphosis angle on MRI. Spine (Phila Pa 1976) 31 : E26-E30, 2006

15. Tousignant M, de Bellefeuille L, O’Donoughue S, Grahovac S : Criterion validity of the cervical range of motion (CROM) goniometer for cervical flexion and extension. Spine (Phila Pa 1976) 25 : 324-330, 2000

16. Tsukimoto $\mathrm{H}$ : On an autopsied case of compression myelopathy with a callus formation in the cervical spinal canal. Nihon-geka-hokan 29 : 1003-1007, 1960

17. Tsuyama $\mathrm{N}$ : Ossification of the posterior longitudinal ligament of the spine. Clin Orthop Relat Res (184) : 71-84, 1984

18. Vedantam R, Lenke LG, Keeney JA, Bridwell KH : Comparison of standing sagittal spinal alignment in asymptomatic adolescents and adults. Spine (Phila Pa 1976) 23 : 211-215, 1998 